\title{
MIRROR SYMMETRY AND QUANTIZATION OF ABELIAN VARIETIES
}

\author{
Yu. I. Manin \\ Max-Planck-Institut für Mathematik, Bonn
}

\section{Introduction}

0. Plan of the paper. This paper consists of two sections discussing various aspects of commutative and non-commutative geometry of tori and abelian varieties.

In the first section, we present a new definition of mirror symmetry for abelian varieties and, more generally, complex and $p$-adic tori, that is, spaces of the form $T / B$ where $T$ is an algebraic group isomorphic to a product of multiplicative groups, $K$ is a complete normed field, and $B \subset T(K)$ is a discrete subgroup of maximal rank in it. We also check its compatibility with other definitions discussed in the literature.

In the second section, we develop an approach to the quantization of abelian varieties first introduced in [Ma1], namely, via theta functions on non-commutative, or quantum, tori endowed with a discrete period lattice. These theta functions satisfy a functional equation which is a generalization of the classical one, in particular, involve a multiplier. Since multipliers cease to be central in the quantum case, one must decide where to put them. In [Ma1] only one-sided multipliers were considered. As a result, the product of two theta functions in general was not a theta function. Here we suggest a partial remedy to this problem by introducing two-sided multipliers. The resulting space of theta functions possesses partial multiplication and has sufficiently rich functorial properties so that rudiments of Mumford's theory $([\mathrm{Mu}])$ can be developed. Main results of [Ma1] are reproduced here in a generalized form, so that this paper can be read independently.

We will now briefly describe a broader picture into which this work fits.

1. On mirror symmetry. In this paper mirror symmetry is understood as a binary relation between (weak) Calabi-Yau manifolds endowed with some additional data. A projective (or compact complex) manifold $V$ is a weak CalabiYau, if it admits nowhere vanishing global volume form. Additional data which are commonly considered are of two types.

A) A symplectic or complexified symplectic structure $\omega_{V}$ on $V$, which is "sufficiently large" in the case of complex base field.

B) A cusp $c_{V}$ in the moduli space (or rather stack) of deformations of $V$, that is, a neighborhood of a point of "maximal degeneration" to which $V$ belongs. 
The mirror partnership relation between $\left(V, c_{V}, \omega_{V}\right)$ and $\left(W, c_{W}, \omega_{W}\right)$ consists in a host of identifications (partly conjectural) of various structures that can be produced starting with such triples. In the case of strict Calabi-Yau's, this includes an identification of two Frobenius manifolds: quantum cohomology of $\left(V, \omega_{V}\right)$ and a germ of the extended moduli space of $W$ with its flat structure determined by $c_{W}$, and similarly with roles of $V$ and $W$ reversed. Generally, one expects also a representation of $V$ and $W$ as dual real Lagrangian torus fibrations over a common base, with a rich structure of Fourier-Mukai transform connecting Lagrangian/complex analytic objects on both sides. For more details on this, see original works [MirS1], [MirS2], [StYZ], [Ko], [Giv], [Bar], [LYZ], and a report [Ma3].

Most important testing ground for all levels of mirror correspondence is furnished by toric mirrors introduced and studied by V. Batyrev. In the simplest version, this construction looks as follows (see [Bat]).

Let $T$ be an $n$-dimensional algebraic torus, that is an algebraic group which is isomorphic to a product of $n$ multiplicative groups $\mathbf{G}_{m}$. It determines (and is functorially determined by any of) the two free abelian groups of rank $n$ : its character group $M_{T}:=\operatorname{Hom}\left(T, \mathbf{G}_{m}\right)$ and its group of one-parametric subgroups $N_{T}:=\operatorname{Hom}\left(\mathbf{G}_{m}, T\right)$. These groups are naturally dual to each other. Denote by $T^{t}$ the dual torus, whose character group is $M_{T^{t}}=N_{T}$ and respectively $N_{T^{t}}=M_{T}$. In the main text of paper, one of these groups is denoted $H$ and another $H^{t}$.

Among various toric compactifications of $T$ we are interested in those for which their anticanonical system is ample. Anticanonical divisors on them are $n$-dimensional Calabi-Yau manifolds.

Such toric compactifications $\bar{T}_{\Delta}$ are naturally indexed by reflexive polyhedra $\Delta$ in $N_{T} \otimes \mathbf{R}$. Via standard convex duality, each such polyhedron determines the dual polyhedron $\Delta^{t}$ in $N_{T^{t}} \otimes \mathbf{R}$ which is also reflexive. According to Batyrev, families of anticanonical hypersurfaces in the respective toric compactifications are expected to be mirror partners:

$$
\bar{T}_{\Delta} \supset\left|-K_{\bar{T}_{\Delta}}\right| \Longleftrightarrow\left|-K_{\bar{T}_{\Delta^{t}}^{t}}\right| \subset \bar{T}_{\Delta^{t}}^{t}
$$

The relevant maximally degenerate CY's are simply divisors at infinity in these toric compactifications.

Supplementing $(T, \Delta)$ with additional combinatorial structure, one can generalize this picture to some Calabi-Yau complete intersections.

The main goal of the first section of this paper is to provide a new definition of mirror symmetry for abelian varieties (and more generally, complex and $p$-adic tori) similar to (0.1). We use a "multiplicative uniformization" which goes back to Jacobi and which represents $\mathcal{A}$ as a quotient of $n$-dimensional algebraic torus $T$ by 
a multiplicative discrete lattice $B \subset T(K)$. Consider now two dual algebraic tori endowed with period lattices which are explicitly identified, that is a diagram of the form:

$$
\left(i, i^{t}\right): T \leftarrow B \rightarrow T^{t}
$$

We will say that pairs $\left(\mathcal{A}:=T(K) / i(B), i^{t}\right)$ and $\left(\mathcal{B}:=T^{t}(K) / i^{t}(B), i\right)$ are mirror dual to each other. Over complex field, we will relate $i^{t}$ (resp. $i$ ) to a structure similar to $\omega_{V}$ (resp. $\omega_{W}$ ) above and compare our mirror relation with that of [Gr1], $[\mathrm{Gr} 2]$, $[\mathrm{AP}]$ and [GolLO]. In particular, we will see how this diagram gives rise to two dual fibrations of the relevant abelian varieties (or complex tori) by mutually dual Lagrangian real tori over the same base.

The choice of multiplicative uniformization is not unique, and it provides the environment for a partial compactification of the relevant moduli space and choice of a maximally degenerate point at the boundary of moduli space to which $\mathcal{A}$ is close, that is, of the relevant cusp of the moduli space. Roughly speaking, at the boundary some generators of the period lattice are forced to vanish so that the rank of the image of $B$ drops. To make it more precise, we can choose a fan $\Phi$ in $N_{T} \otimes \mathbf{R}$, construct the dual fan $\Phi^{t}$ and study the moduli space of the refined diagrams

$$
\left(i, i^{t}\right): \bar{T}_{\Phi}(K) \leftarrow B \rightarrow \bar{T}_{\Phi^{t}}^{t}(K)
$$

In this context, it turns out that the fibers of the dual mirror fibrations lie in the monodromy invariant homology class $\tau$ of middle dimension, as in the strict Calabi-Yau case.

0.3. Commutative and non-commutative tori and theta functions. In the second section of this paper we address the problem of constructing "quantum abelian varieties". As we already mentioned, what we actually construct is a linear space of quantized theta functions (on a noncommutative torus with a period lattice) endowed with partial multiplication. We regard this semiring as a quantum deformation of the universal multigraded ring $\oplus \Gamma(L), L \in \operatorname{Pic} \mathcal{A},[-1]^{*}(L) \cong L$, which makes sense for any abelian variety $\mathcal{A}$ (or indeed for any projective variety).

Since the whole construction is algebraic, it can be performed over any complete normed field, for example, $p$-adic field, and applied to the classical abelian varieties as well. Only those $p$-adic abelian varieties admit a multiplicative uniformization which have maximally degenerate stable reduction modulo $p$. This is the definition of the $p$-adic cusp. Diagrams of the type (0.2) make full sense in this context as well, but $i^{t}$ admits no straightforward interpretation as anything like symplectic form. It would be interesting to investigate the meaning of $p$-adic $B$-fields for strict Calabi-Yau manifolds. 
We will now briefly discuss issues of non-commutative geometry involved in our construction of quantized theta functions. The standard approach is via deformation of classical function rings, and this intuition guided our initial construction in [Ma1] and to a certain degree its extension presented in this paper.

A complementary paradigm, made explicit on many occasions in Connes' papers and the book $[\mathrm{Co}]$ is the natural appearance of non-commutative rings as objects encoding commutative spaces "with bad geometric properties", typically quotients of commutative spaces by non-separated equivalence relations.

For example, in the context of multiplicative uniformization of abelian varieties $\mathcal{A}(\mathbf{C})=T(\mathbf{C}) / B$, the multiplicative period lattice $B$ can degenerate also by ceasing to be discrete, although keeping its rank constant, and in this case it becomes natural to interpret $T(\mathbf{C}) / i(B)$ in the realm of non-commutative geometry.

To be concrete, consider the case of one-dimensional commutative torus $T$. Its maximal toric compactification is $\mathbf{P}^{1}=\mathbf{G}_{m} \cup\{0\} \cup\{\infty\}$. Choose $B=\mathbf{Z}$ so that $\mathcal{A}=\mathbf{C}^{*} /\left(q^{\mathbf{Z}}\right)$. On the level of diagram $(0.3)$ we can choose here any $q \in \mathbf{P}^{1}(\mathbf{C})$. When $|q| \neq 0,1, \infty$, we get an elliptic curve $\mathcal{A}=E_{q}$ fibered by images of real tori $|z|=$ const (where $z$ is the coordinate on $\mathbf{C}^{*}$ ) over the circle $\mathbf{R} / \mathbf{Z} \log |q|$. Dualizing this fibration, we will get the mirror dual elliptic curve. The points $0, \infty$ become divisors on a modular curve and provide the familiar degeneration picture in algebraic geometry. Points where $q$ is a root of unity also become visible in algebraic geometry as cusp points of modular curves of higher levels. However, $q$ of infinite order with $|q|=1$ are not considered in algebraic geometry at all. One way to interprete the space $\mathbf{G}_{m} /\left(q^{\mathbf{Z}}\right)$ in this case is to identify it with (one of the versions of) the two-dimensional non-commutative torus $T_{q}$, whose function ring is generated by $x^{ \pm 1}, y^{ \pm 1}$ satisfying the commutation relation $x y=q y x$. See Appendix for an informal explanation of this in the context of noncommutative geometry à la Alain Connes.

One remarkable trace of this origin of $T_{q}$ for $q=e^{2 \pi i \tau}, \tau \in \mathbf{R}$, as a limiting elliptic curve is the re-appearance of the modular group $S L(2, Z)$ as a symmetry group in the non-commutative situation: acting on $\tau$, it produces Morita equivalent function rings of $T_{q}$ 's. See [RiS] for a thorough discussion of this in arbitrary dimension.

What I want to stress here, is a somewhat neglected complementary aspect of this picture: namely, that even if the group $q^{\mathbf{Z}}$ is discrete, $T_{q}$ still can be viewed as a legitimate incarnation of the elliptic curve $E_{q}$ in the non-commutative world. A systematic treatment of the correspondence between, say, coherent sheaves on $E_{q}$ and modules over $T_{q}$ remains a problem for future, but see the recent preprint [BEG] for some precise facts about this correspondence, and many interesting suggestions are contained in [So].

This remark throws some light on the problems left open with our approach to 
quantized theta-functions, for example, that of functional equations corresponding to a change of cusp. Some of our non-commutative abelian varieties $T(H, \alpha) / B$ where $T(H, \alpha)$ is a non-commutative torus with quantization parameter $\alpha$ (see 2.1) can be understood as a result of taking a quotient of a commutative torus $T\left(H^{\prime}, 1\right)$ of halved rank, by a non-discrete period subgroup with too many generators. This might provide a bridge between our construction and that of Weinstein ([We]) remaining entirely in the realm of commutative geometry.

Acknowledgement. I am grateful to D. Orlov for illuminating correspondence on Abelian mirrors and comments on the paper [GolLO]. I learned from Y. Soibelman the philosophy of treating boundaries of various moduli spaces as bridges to the non-commutative realm. A. Polishchuk has drawn my attention to the paper $[\mathrm{Fu}]$ and sent me a copy of it.

\section{Mirror symmetry for complex tori and abelian varieties}

1.1. Toric formalism. Since we will have to work with several different types of tori which must be carefully distinguished, we start with some terminological conventions.

Let $K$ be a ground field, $H$ a free abelian group of rank $n$ which we will always write additively. Algebraic torus $T$ with character group $H$ is the affine spectrum of the group ring of $H$, and $H=\operatorname{Hom}\left(T, \mathbf{G}_{m}\right)$. Put $H^{t}:=\operatorname{Hom}\left(\mathbf{G}_{m}, T\right)$. Groups $H, H^{t}$ are connected by the canonical duality map $H \times H^{t} \rightarrow$ Aut $\mathbf{G}_{m}=\mathbf{Z}$.

An element $h \in H$ considered as a character of $T$ will be denoted $e(h)$ ( $e$ for exponential). We have $e\left(h+h^{\prime}\right)=e(h) e\left(h^{\prime}\right)$.

In the next section, we will consider noncommutative algebraic tori as well, for which the multiplication rule for $e(h)$ is twisted: see (2.3) below.

1.1.1. Duality of algebraic tori. If $T$ is an algebraic torus with character group $H_{T}$, the dual algebraic torus $T^{t}$ has the character group $H_{T^{t}}:=H_{T}^{t}=$ $\operatorname{Hom}\left(H_{T}, \mathbf{Z}\right)$.

1.1.2. Periods and abstract tori. Let $H$ be the character group of $T$. Denote by $B$ another free abelian group of the same rank $n$, and by $i: B \rightarrow T(K)$ a homomorphism which we will call "period map". Since $T(K)=\operatorname{Hom}\left(H, K^{*}\right)$, to give $i$ is the same as to give a pairing $H \times B \rightarrow K^{*}$ such that $(h, b)$ is the value of $e(h)$ at the point $i(b)$.

We will refer to the quotient space $T / i(B)$ as an abstract torus, and to $T$ as its covering algebraic torus. One may imagine $T / i(B)$ simply as a functor of points on $K$-algebras $R \mapsto T(R) / i(B)$. In the case when $K=\mathbf{C}$ and $i$ is an injection with discrete image, $T(\mathbf{C}) / i(B)$ is a complex torus, which may admit a structure of abelian variety (it is then unique). However, a large part of our elementary 
formalism will not depend on additional assumptions. Thus, as explained in the Introduction, we will be able to include into our mirror picture $p$-adic tori and abelian varieties, and eventually non-commutative tori viewed as models of $T(\mathbf{C}) / i(B)$ in non-commutative geometry.

In order not preclude the eventual interpretation of the space $\mathcal{A}=T / i(B)$ we will consistently identify $\mathcal{A}$ with the triple

$$
\left(H_{\mathcal{A}}, B_{\mathcal{A}},(,)_{\mathcal{A}}: H_{\mathcal{A}} \times B_{\mathcal{A}} \rightarrow K^{*}\right)
$$

as above.

1.1.3. Poincaré dual abstract tori. By definition, Poincaré duality interchanges characters and periods. More precisely, $\mathcal{A}$ and $\widehat{\mathcal{A}}$ are Poincaré dual if

$$
H_{\widehat{\mathcal{A}}}=B_{\mathcal{A}}, B_{\widehat{\mathcal{A}}}=H_{\mathcal{A}},(b, h)_{\widehat{\mathcal{A}}}=(h, b)_{\mathcal{A}}^{-1} \text { for } h \in H_{\mathcal{A}}, b \in B_{\mathcal{A}} .
$$

For abelian varieties this agrees with the classical definition. Notice however, that a choice of the covering algebraic torus is an additional structure, and we explicitly extend Poincaré duality to this context.

1.1.4. Framed tori. A framing of the abstract torus (1.1) is a map $i^{t}: B_{\mathcal{A}} \rightarrow$ $T_{\mathcal{A}}^{t}(K)$. A framed abstract torus is a pair $\left(\mathcal{A}, i^{t}\right)$.

A framing of the complex torus or abelian variety consists of its representation as an abstract torus and framing of that abstract torus.

1.1.5. Mirror dual framed abstract tori. Two framed abstract tori $\left(\mathcal{A}, i^{t}\right)$ and $(\mathcal{B}, i)$ are called mirror dual, or mirror partners, if their covering algebraic tori are dual, and their periods are explicitly identified. More precisely, the relation of mirror partnership is provided by diagrams of the form $(0.2)$. If one thinks about $i, i^{t}$ in terms of the respective character/period pairings (1.1), the mirror duality is provided by pairings

$$
H_{\mathcal{A}} \times B_{\mathcal{A}} \rightarrow K^{*}, H_{\mathcal{A}}^{t} \times B_{\mathcal{A}} \rightarrow K^{*}
$$

A framing is called non-degenerate if both kernels of the respective pairing are trivial.

This notion of mirror duality is the main definition of this section. We start with studying it for $K=\mathbf{C}$.

1.2. Complex tori. Assume that $i(B)$ is discrete in $T(\mathbf{C})$. Put

$$
\Gamma=\Gamma_{\mathbf{Z}}=\pi_{1}(T(\mathbf{C}) / i(B), 0)=H_{1}(\mathcal{A}, \mathbf{Z})
$$


$\Gamma_{\mathbf{R}}=\Gamma_{\mathbf{Z}} \otimes \mathbf{R}$ and similarly for $\Gamma_{\mathbf{C}}$. If $T(\mathbf{C}) / i(B)$ is denoted $\mathcal{A}$, we may write $\Gamma_{\mathcal{A}}$, $\Gamma_{\mathcal{A}, \mathbf{R}}$, etc.

The real space $\Gamma_{\mathbf{R}}$ can be identified with the Lie algebra of $\mathcal{A}$, and the exponential map exp : $\Gamma_{\mathbf{R}} \rightarrow \mathcal{A}$ with kernel $\Gamma_{\mathbf{Z}}$ is the universal covering of $\mathcal{A}$.

Let $H=H_{\mathcal{A}}$ be the character group of $T$. The map $h \mapsto \frac{1}{2 \pi i} \frac{d e(h)}{e(h)}$ induces canonical identification $H=H^{1}(T(\mathbf{C}), \mathbf{Z})$. Hence we have an exact sequence

$$
0 \rightarrow H_{\mathcal{A}}^{t} \rightarrow H_{1}(\mathcal{A}, \mathbf{Z}) \rightarrow B_{\mathcal{A}} \rightarrow 0
$$

where the third arrow is induced by $i$. Similarly, we have for a mirror dual framed torus $\left(\mathcal{B}=T^{t}(\mathbf{C}) / i^{t}(B), i\right)$

$$
0 \rightarrow H_{\mathcal{A}} \rightarrow H_{1}(\mathcal{B}, \mathbf{Z}) \rightarrow B_{\mathcal{A}} \rightarrow 0
$$

where the third arrow is now induced by $i^{t}$.

1.2.1. Mirror partners as dual real torus fibrations. In this subsection we will show that our definition of mirror partners $(\mathcal{A}, \mathcal{B})$ over $\mathbf{C}$ naturally fits into the general context of Lagrangian/complex duality: see [AP], [StYZ], [Gr2].

We start with a brief description of this context.

Let $(X, \omega)$ be a $C^{\infty}$ symplectic manifold, endowed with a submersion $p_{X}: X \rightarrow$ $U$ whose fibers are Lagrangian tori. We will fix also a Lagrangian section $0_{X}: U \rightarrow$ $X$.

Using $\omega$, we can identify the bundle of Lie algebras of the tori $p_{X}^{-1}(u), u \in U$, with the cotangent bundle $T_{U}^{*}$. Hence we have a canonical isomorphism $X=T_{U}^{*} / H$ where $H$ is a Lagrangian sublattice in $T_{U}^{*}$ with respect to the lift of $\omega$ which is the standard symplectic form on the cotangent bundle. There exists also a canonical flat symmetric connection on $T_{U}^{*}$ for which $H$ is horizontal.

The local system $H^{t}=\mathcal{H o m}(H, \mathbf{Z})$ is embedded as a sublattice into $T_{U}$, and we can define the mirror partner of $\left(p_{X}: X \rightarrow U, \omega, 0_{X}\right)$ as the toric fibration $Y:=T_{U} / H^{t}$ endowed with the projection to the same base $p_{Y}: Y \rightarrow U$ and the zero section $0_{Y}$.

Passing from $X$ to $Y$ we have lost the symplectic form. To compensate for this loss, we have acquired a complex structure $J: T_{Y} \rightarrow T_{Y}$ which can be produced from $\left(p: X \rightarrow U, \omega, 0_{X}\right)$ in the following way. The flat connection on $T_{U}$ obtained by the dualization from $T_{U}^{*}$ produces a natural splitting $T_{Y}=p_{Y}^{*}\left(T_{U}\right) \oplus p_{Y}^{*}\left(T_{U}\right)$. With respect to this splitting, $J$ acts as $\left(t_{1}, t_{2}\right) \mapsto\left(-t_{2}, t_{1}\right)$.

Conversely, suppose that we have a complex manifold $Y$ endowed with a fibration by real tori $Y \rightarrow U$ with zero section, such that the operator of complex structure 
along the zero section identifies $T_{U}$ with the bundle of Lie algebras of fibers. Then we can consecutively construct the lattice $H^{t} \subset T_{U}$, the dual fibration $X:=T_{U}^{*} / H$ and the symplectic form on $X$ coming from the cotangent bundle.

Now we can return to complex tori.

Put $S^{1}=\{|z|=1 \mid z \in \mathbf{C}\}$. We have the Lie group isomorphism $\mathbf{C}^{*} \rightarrow S^{1} \times \mathbf{R}$ : $z \mapsto(z /|z|, \log |z|)$. This induces an isomorphism

$$
(\alpha, \lambda): T(\mathbf{C}) \rightarrow \operatorname{Hom}\left(H, S^{1}\right) \times \operatorname{Hom}(H, \mathbf{R}) .
$$

If $i(B)$ is discrete of maximal rank which $\mathrm{I}$ will assume, then $\lambda \circ i(B)$ is an additive lattice in the real space $\operatorname{Hom}(H, \mathbf{R})$. Thus (1.6) produces a real torus fibration of $T(\mathbf{C})$ over the base which is as well a real torus of the same dimension:

$$
0 \rightarrow \operatorname{Hom}\left(H, S^{1}\right) \rightarrow T(\mathbf{C}) / i(B) \rightarrow \operatorname{Hom}(H, \mathbf{R}) / \lambda \circ i(B) \rightarrow 0 .
$$

Similarly, we have

$$
0 \rightarrow \operatorname{Hom}\left(H^{t}, S^{1}\right) \rightarrow T^{t}(\mathbf{C}) / i^{t}(B) \rightarrow \operatorname{Hom}\left(H^{t}, \mathbf{R}\right) / \lambda^{t} \circ i^{t}(B) \rightarrow 0
$$

where $\lambda^{t}$ is defined for $T^{t}$ in the same way as $\lambda$ for $T$. Let us identify linear real spaces $H_{\mathbf{R}}$ with $H_{\mathbf{R}}^{t}$ in such a way that lattice points $\lambda \circ i(b)$ and $\lambda^{t} \circ i^{t}(b)$ are identified for all $b \in B$. Then (1.7) and (1.8) become dual real torus fibrations over the common base.

The relevant complex structures in our context come from covering tori. We have to introduce symplectic forms. Let us construct, say, $\omega_{\mathcal{A}}$. From (1.8) one sees that $\mathcal{A}=T(\mathbf{C}) / i(B)$ can be obtained as quotient space of the tangent bundle of the base by a lattice. The tangent bundle (and the lattice) is canonically trivialized, and its fiber is $H_{\mathbf{R}}$. Using the two framings, we have identified $H_{\mathbf{R}}$ with $H_{\mathbf{R}}^{t}$, that is, tangent bundle with cotangent bundle. The canonical symplectic form on the cotangent bundle becomes our $\omega_{\mathcal{A}}$. Clearly, fibers of (1.7) are Lagrangian tori. It remains to check that $\omega_{\mathcal{A}}$ determines $I_{\mathcal{B}}$ as above, but this is quite straightforward.

1.2.2. Maximal degeneration point and monodromy. Let us consider now the situation, described by (one half of) the diagram (0.3). More precisely, consider the space of maps of $B$ to a neighbourhood of a point of maximal degeneration in some toric compactification $\bar{T}_{\Phi}$. Such a point is a zero-dimensional orbit of $T$, thus it corresponds to a maximal cone in $\Phi$. Assume for simplicity that it is the simplicial cone generated by a basis of $H^{t}$. This means that we identify $H^{t}$ with $\mathbf{Z}^{n}, T(\mathbf{C})$ with $\left(\mathbf{C}^{*}\right)^{n}$, and choose as partial compactification the imbedding $\left(\mathbf{C}^{*}\right)^{n} \subset \mathbf{C}^{n}$. Let $D_{r}$ be the $r$-th coordinate hyperplane in $\mathbf{C}^{n}$.

Choosing a basis of $B$ as well, that is, identifying it with $\mathbf{Z}^{n}$, we see that the region of the partially compactified moduli space of multiplicatively uniformized 
complex tori that we are interested in can be identified with an open subspace of the matrix space $\mathbf{C}^{n \times n}$ whose columns generate a multiplicative sublattice. The discriminant locus in this region consists of its intersection with $\cup D_{r s}$ where $D_{r s}$ is the $r$-th coordinate hyperplane in the $s$-th copy of $\mathbf{C}^{n}$ times other copies. The origin (intersection of all $D_{r s}$ ) is the maximum degeneration point.

Let $q=\left(q_{r s}\right)$ be a point of the moduli space, $\mathcal{A}_{q}$ the respective torus. Denote by $\gamma_{r} \in H_{1}\left(\mathcal{A}_{q}, \mathbf{Z}\right)$ the image of the $r$-th $S^{1}$ in (1.7) with counterclockwise orientation. Let $M_{r s}$ be the monodromy action of a small counterclockwise loop around $D_{r s}$ in the moduli space. All cycles $\gamma_{r}$ are monodromy invariant. Let $\beta_{s}$ be any lift to $H_{1}\left(\mathcal{A}_{q}, \mathbf{Z}\right)$ of the $s$-th 1 -cycle in the base torus in (1.7). Then $M_{r s}$ transforms $\beta_{s}$ into $\beta_{s}+\gamma_{r}$ and leaves other 1 -cycles invariant.

Thus, the homology class $\tau$ of any fiber of (1.7) generates the cyclic group of invariant cycles of middle dimension.

This statement holds independently of the choice of the simplicial fan and a maximal degenerating cone in it. In this sense, the choice of a covering torus alone encodes essential information about large complex structure.

1.3. Framings and well-becoming pairs. In this subsection we will compare our construction of mirror partners with that of [GolLO]. In that paper, the additional structure on $\mathcal{A}$ is a complex-valued 2 -form $\omega$ rather than symplectic form as above. I will show that this as well can be related to an appropriate framing.

Consider first a pair of framed complex tori $\left(\mathcal{A}, i^{t}\right)$ and $(\mathcal{B}, i)$ which are mirror partners as above. In [GolLO], sec. 10, the authors use decompositions

$$
\Gamma_{\mathcal{A}}=\Gamma_{1, \mathcal{A}} \oplus \Gamma_{2, \mathcal{A}}, \Gamma_{\mathcal{B}}=\Gamma_{1, \mathcal{B}} \oplus \Gamma_{2, \mathcal{B}}
$$

We will call such decompositions compatible with our choice of covering tori, if $\Gamma_{1, \mathcal{A}}=H_{\mathcal{A}}^{t}, \Gamma_{1, \mathcal{B}}=H_{\mathcal{A}}$ as in (1.4), (1.5). Thus compatible decompositions are simply splittings of (1.4) and (1.5). The spaces $\Gamma_{\mathcal{A}, \mathbf{R}}, \Gamma_{\mathcal{B}, \mathbf{R}}$ are endowed respectively with complex structures $I_{\mathcal{A}}, I_{\mathcal{B}}$ coming from covering tori. Clearly,

$$
\Gamma_{\mathcal{A}, \mathbf{R}}=H_{\mathcal{A}, \mathbf{R}}^{t} \oplus I_{\mathcal{A}} H_{\mathcal{A}, \mathbf{R}}^{t}
$$

so that compatible splittings satisfy the condition 10.3.1 (2) of [GolLO].

Consider now only $\mathcal{A}$, but equipped with a class $\omega \in H^{2}(\mathcal{A}, \mathbf{C})$ interpreted as an antisymmetric complex-valued form on $\Gamma_{\mathcal{A}}$. Assume that the extension of $\omega$ to $\Gamma_{\mathcal{A}, \mathbf{R}}$ is $I_{\mathcal{A}}$-invariant (see [GolLO], last lines of 1.4 for an explanation of this condition). Assume moreover that there exists a compatible decomposition of $\Gamma_{\mathcal{A}}$ such that $\Gamma_{1, \mathcal{A}}$ and $\Gamma_{2, \mathcal{A}}$ are $\omega$-isotropic. This means that $(\mathcal{A}, \omega)$ is a well-becoming pair in the sense of [GolLO], 10.3.1. Looking at (1.4), we see that $\omega$ can be uniquely reconstructed from its restriction which we also denote $\omega$

$$
\omega: H_{\mathcal{A}}^{t} \times B_{\mathcal{A}} \rightarrow \mathbf{C}
$$


Exponentiating (1.9) we produce a framing $i^{t}: B \rightarrow T^{t}(\mathbf{C})$ in the form (1.3), that is

$$
e(h)\left(i^{t}(b)\right)=e^{2 \pi i \omega\left(h, b+H_{\mathcal{A}}^{t}\right)}, h \in H_{\mathcal{A}}^{t}, b \in B .
$$

Clearly, $i^{t}$ remains the same, if we choose another compatible isotropic splitting. Moreover, it does not change if we add to $\omega$ another pairing taking values in $2 \pi i \mathbf{Z}$.

In this way we get a map from the set of all well-becoming pairs $(\mathcal{A}, \omega)$ admitting compatible isotropic splittings in the sense of [GolLO] to the set of framed abstract tori $\left(\mathcal{A}, i^{t}\right)$ with non-degenerate framings in our sense.

We can now complete the comparison of our definition of mirror duality with that of [GolLO].

1.3.1. Theorem. Let $\left(\mathcal{A}, i^{t}\right),(\mathcal{B}, i)$ be a mirror dual pair of framed complex abstract tori, admitting lifts $\left(\mathcal{A}, \omega_{\mathcal{A}}\right),\left(\mathcal{B}, \omega_{\mathcal{B}}\right)$ to well-becoming pairs. Then $\left(\mathcal{A}, \omega_{\mathcal{A}}\right)$, $\left(\mathcal{B}, \omega_{\mathcal{B}}\right)$ are mirror dual in the sense of [GolLO].

Proof. We will compare our setting with that of [GolLO], 10.4 and 10.4.1. Groups $\Gamma_{1}, \Gamma_{2}$ in [GolLO] are our $H_{\mathcal{A}}^{t}, B$, fundamental group of the mirror dual torus is $\Gamma_{1}^{t} \oplus \Gamma_{2}$. This means that the real covering torus of their $\mathcal{B}$ is the same as ours, that is $T^{t}$. It remains to compare the complex structures. In our case it is simply induced from $T^{t}(\mathbf{C})$. In [GolLO] it is described with the help of the complex structure operator $I$ acting upon $\left(\Gamma_{1}^{t} \oplus \Gamma_{2}\right) \otimes \mathbf{R}$ produced from $\omega$ in two steps: via formula (14) and subsequent projection described in 10.4.

In order to check that they coincide, we will reproduce a part of the argument in [GolLO] in the form which hopefully clarifies the meaning of their crucial formula (14).

Since the following construction must be considered in two different situations, described in [GolLO] 9.2 and 10.4 respectively, we slightly change the scope of our notation. From now on, $\Gamma_{1}, \Gamma_{2}$ will denote two abstract free abelian groups of the same finite rank, $\Gamma=\Gamma_{1} \oplus \Gamma_{2}, \Gamma^{\prime}=\Gamma_{1}^{t} \oplus \Gamma_{2}$. Introduce two real tori

$$
\mathcal{C}=\left(\Gamma_{1} \oplus \Gamma_{2}\right)_{\mathbf{R}} / \Gamma_{1} \oplus \Gamma_{2}, \mathcal{C}^{\prime}=\left(\Gamma_{1}^{t} \oplus \Gamma_{2}\right)_{\mathbf{R}} / \Gamma_{1}^{t} \oplus \Gamma_{2}
$$

Consider the data of two types.

(i) Complex structures on $\mathcal{C}^{\prime}$ described by the operators $I$ on $\left(\Gamma_{1}^{t} \oplus \Gamma_{2}\right)_{\mathbf{R}}$ such that their crossover components

$$
I_{12}: \Gamma_{2, \mathbf{R}} \rightarrow \Gamma_{1, \mathbf{R}}^{t}, I_{21}: \Gamma_{1, \mathbf{R}}^{t} \rightarrow \Gamma_{2, \mathbf{R}}
$$

are bijective.

(ii) Forms $\omega \in \wedge^{2} \Gamma_{\mathbf{C}}^{t}$ for which $\Gamma_{1, \mathbf{C}}$ and $\Gamma_{2, \mathbf{C}}$ are maximal isotropic. 
We will establish a bijection between them in the following way.

Let us start with a complex structure $I$ in $\mathcal{C}^{\prime}$. It determines (and is determined by) the space of invariant holomorphic 1 -forms on $\mathcal{C}^{\prime}$. Integrating them over $\Gamma_{1}^{t} \subset$ $H_{1}\left(\mathcal{C}^{\prime}, \mathbf{Z}\right)$, we will get all additive maps $\Gamma_{1}^{t} \rightarrow \mathbf{C}$, in particular, all elements of $\Gamma_{1}$. So we have an embedding $\Gamma_{1} \rightarrow H^{0}\left(\mathcal{C}^{\prime}, \Omega^{1}\right): \gamma \rightarrow \nu_{\gamma}$ such that for all $\beta \in \Gamma_{1}^{t}, \gamma \in \Gamma_{1}$,

$$
(\beta, \gamma)=\int_{\beta} \nu_{\gamma}
$$

This allows one to define a non-degenerate scalar product $\langle\rangle:, \Gamma_{2} \otimes \Gamma_{1} \rightarrow \mathbf{C}$ :

$$
\left\langle\gamma_{2}, \gamma_{1}\right\rangle=\int_{\gamma_{2}} \nu_{\gamma_{1}}
$$

Finally, we can extend it to a complex skew-symmetric form $\omega$ on $\Gamma$ declaring $\Gamma_{1}$ and $\Gamma_{2}$ to be isotropic:

$$
\omega\left(\left(\gamma_{1}, \gamma_{2}\right),\left(\gamma_{1}^{\prime}, \gamma_{2}^{\prime}\right)\right)=\left\langle\gamma_{2}, \gamma_{1}^{\prime}\right\rangle-\left\langle\gamma_{2}^{\prime}, \gamma_{1}\right\rangle
$$

If we choose a basis of $\Gamma_{1}^{t}, \Gamma_{2}$ and a basis of holomorphic 1 -forms whose period matrix over $\Gamma_{1}^{t}$ is the identity $E$, then the Gram matrix of the pairing $\langle$,$\rangle will be$ just the second half of the total period matrix. Let us denote it $\tau$. Then $e^{2 \pi i \tau}$ is the matrix generating the multiplicative period lattice in the covering complex torus $\Gamma_{\mathbf{C}}^{\prime} / \Gamma_{1}^{t}$ which in view of (1.14) agrees with (1.10).

Arguing now in reverse direction, we will show that knowing $\tau$ we can reconstruct the operator $I$ in the same basis and get essentially the [GolLO] formula. In fact, $I$ is uniquely determined by the requirement that for all $\gamma \in \Gamma^{\prime}$ and all holomorphic $\nu$ we have $\int_{I \gamma} \nu=i \int_{\gamma} \nu$. Hence to find $I$ we must solve the matrix system

$$
(\operatorname{Re} \tau+i \operatorname{Im} \tau, E)\left(\begin{array}{cc}
X & Y \\
U & V
\end{array}\right)=(-\operatorname{Im} \tau+i \operatorname{Re} \tau, i E)
$$

which gives

$$
I=\left(\begin{array}{ll}
X & Y \\
U & V
\end{array}\right)=\left(\begin{array}{cc}
(\operatorname{Im} \tau)^{-1} \operatorname{Re} \tau & (\operatorname{Im} \tau)^{-1} \\
-\operatorname{Im} \tau-\operatorname{Re} \tau(\operatorname{Im} \tau)^{-1} \operatorname{Re} \tau & -\operatorname{Re} \tau(\operatorname{Im} \tau)^{-1}
\end{array}\right)
$$

For the first application of (1.15), let us choose a real torus $\mathcal{A}$ with period lattice $\Gamma_{\mathcal{A}}$ and put $\mathcal{C}=\mathcal{A} \oplus \widehat{\mathcal{A}}$ where $\widehat{\mathcal{A}}$ is the Poincaré dual real torus, that is $\Gamma_{\widehat{\mathcal{A}}}=\Gamma_{\mathcal{A}}^{t}$ (this does not contradict our multiplicative description (1.2) although it may not be immediately obvious). Put $\Gamma_{1}=\Gamma_{\mathcal{A}}, \Gamma_{2}=\Gamma_{\mathcal{A}}^{t}$. Comparing our formula (1.15) 
with [GolLO] (14) in this situation, we see that our $I$ coincides with their $I_{\omega}$. In [GolLO], 8.4, $\mathcal{A}$ additionally possesses a complex structure, which produces the canonical complex structures on $\widehat{\mathcal{A}}, \mathcal{C}$ and $\mathcal{C}^{\prime}$, say, $J$. Moreover, $\omega$ is restricted to lie in the complexified Néron-Severi group, and as a result $I$ commutes with the inherited complex structure $J$ on $\mathcal{C}^{\prime}$.

The setup which we are discussing in the Theorem 1.3.1 is that of [GolLO] 10.4 and 10.5. The relevant torus $\mathcal{C}$ is now $\mathcal{A}$, and its homology lattice is now split by the choice of a compatible decomposition like in 1.3. Extension of this splitting to $\mathcal{A} \oplus \widehat{\mathcal{A}}$ produces the period matrix $\tau$ which is block diagonal and consists of two blocks. Formula (1.15) is still valid for the mirror complex structure, when one replaces $\tau$ in it by the respective block. Putting everything together we see that our complex structure determined essentially by (1.10) indeed agrees with that of [GolLO].

1.3.2. Remark. [GolLO] contains several tentative descriptions of mirror dual pairs differing mostly by the exact choice of the additional structure that should be added to $\mathcal{A}$. Our Theorem 1.3.1 together with the Theorem 10.5 in [GolLO] indicates that the notion of a well-becoming pair endowed with a choice of one half of an isotropic decomposition captures just right amount of information. Replacing this structure by that of framing, we make explicit the important aspect of "large complex structure" in the case $K=\mathbf{C}$ and simultaneously extend the definition to abstract tori over arbitrary fields.

\section{Quantized theta-functions and abelian varieties.}

2.1. Category of non-commutative tori. Let $H$ be a free abelian group of finite rank and $\alpha: H \times H \rightarrow K^{*}$ an alternating pairing: for all $h, g \in H$

$$
\alpha(h, g)=\alpha(g, h)^{-1}, \quad \alpha\left(h_{1}+h_{2}, g\right)=\alpha\left(h_{1}, g\right) \alpha\left(h_{2}, g\right) .
$$

A morphism $f:\left(H_{1}, \alpha_{1}\right) \rightarrow\left(H_{2}, \alpha_{2}\right)$ is a group homomorphism $f: H_{1} \rightarrow H_{2}$ such that for all $h, g \in H_{1}$ we have

$$
\alpha_{2}^{2}(f(h), f(g))=\alpha_{1}^{2}(h, g) .
$$

The bilinear form

$$
\varepsilon_{f}(h, g):=\alpha_{1}(h, g) \alpha_{2}^{-1}(f(h), f(g))
$$

with values in $\{ \pm 1\}$ is called the characteristic of $f$ (and of $F$ ).

Any such pair $(H, \alpha)$ will be called the character group of the non-commutative torus $T(H, \alpha)$ whose ring of algebraic functions $A l(H, \alpha)$ is the linear space spanned over $K$ by the symbols $e(h), h \in H$, with multiplication rule

$$
e(h) e\left(h^{\prime}\right)=\alpha\left(h, h^{\prime}\right) e\left(h+h^{\prime}\right)
$$


We may write $e_{H, \alpha}(h)$ for $e(h)$ if need be.

Notice that $\varepsilon(h):=\alpha(h, h)$ is a character of $H$ taking values \pm 1 , and that from (2.3) we get the following formulas:

$$
\begin{gathered}
e\left(h_{1}\right) e\left(h_{2}\right) e\left(h_{3}\right)=\alpha\left(h_{1}, h_{2}\right) \alpha\left(h_{1}, h_{3}\right) \alpha\left(h_{2}, h_{3}\right) e\left(h_{1}+h_{2}+h_{3}\right), \\
e(h)^{-1}=\varepsilon(h) e(-h) .
\end{gathered}
$$

We can also consider the two-sided $A l(H, \alpha)$-module of formal functions $A f(H, \alpha)$ consisting of infinite linear combinations $\sum_{h} a_{h} e(h), a_{h} \in K$, and, in the case of a complete normed field $K$ and an unitary quantization parameter $\alpha$ (that is, $|\alpha|=1)$ the ring of analytic functions $A n(H, \alpha)$ consisting of those formal functions for which $\left|a_{h}\right|\|h\|^{N} \rightarrow 0$ for any $N$ as $\|h\| \rightarrow \infty,\|h\|$ being any Euclidean norm on $H$.

The form $\alpha$ can be called the quantization parameter. When $\alpha \equiv 1$, we get the usual notions of commutative geometry, so that $T(H, 1)$ is the algebraic torus with character group $H$.

$A$ morphism $F: T(H, \alpha) \rightarrow T\left(H^{\prime}, \alpha^{\prime}\right)$, by definition, is given by the contravariant $K$-algebra homomorphism $F^{*}: A l\left(H^{\prime}, \alpha^{\prime}\right) \rightarrow A l(H, \alpha)$.

2.1.1. Proposition. a) The set of invertible elements of $A l(H, \alpha)$ is $\{$ a e $(h) \mid a \in$ $\left.K^{*}, h \in H\right\}$. If $F: T\left(H_{2}, \alpha_{2}\right) \rightarrow T\left(H_{1}, \alpha_{1}\right)$ is a morphism of non-commutative tori, then the induced map $f=[F]: H_{1} \rightarrow H_{2}$ determined by $F^{*}(e(h))=$ $a_{h} e(f(h)), a_{h} \in K^{*}$, is additive and satisfies (2.2) and thus is a morphism of character groups.

b) The set of all morphisms $F$ with fixed $f=[F]$ is either empty, or has a natural structure of the principal homogeneous space over the group $T\left(H_{1}, 1\right)(K)=$ $\operatorname{Hom}\left(H_{1}, K^{*}\right)$. In particular, if the characteristic of $f$ is 1 , then $F^{*}: e(h) \mapsto$ $e(f(h))$ extends to a uniquely defined morphism of rings of algebraic functions.

c) Any morphism $F^{*}$ extends to Af by $F^{*}\left(\sum a_{h} e(h)\right)=\sum a_{h} F^{*}(e(h))$. If $K$ is normed and $\alpha$ unitary, then this extension maps analytic functions to analytic.

Proof. The first statement follows from the fact that $H$ can be endowed with the structure of a well-ordered group. For any such structure, the highest (resp. lowest) terms of a product are products of the highest (resp. lowest) terms, so that an invertible element coincides with its highest and lowest term.

To prove the second statement, rewrite the equality

$$
F^{*}(e(h) e(g))=F^{*}(e(h)) F^{*}(e(g))
$$

using (2.3). Comparing the $e$-terms, we see that $f(h+g)=f(h)+f(g)$. Comparing the coefficients, we get

$$
a_{h} a_{g} a_{h+g}^{-1}=\alpha_{1}(h, g) \alpha_{2}^{-1}(f(h), f(g)) .
$$


The left hand side is a symmetric form in $h, g$, whereas the right hand side is alternate. Therefore this form takes values $\{ \pm 1\}$. Hence (2.2) holds, and (2.6) is the characteristic $\varepsilon_{f}(h, g)$ of $f$.

Finally, let $f$ be a morphism of character groups with characteristic $\varepsilon$. Then ring morphisms $F^{*}$ with $[F]=f$ bijectively correspond to the solutions $\left\{a_{h} \mid h \in H_{1}\right\}$ of the equations $a_{h} a_{g} a_{h+g}^{-1}=\varepsilon_{f}(h, g)$. If one such solution exists, then all others are of the form $a_{h} c(h)$ where $c: H_{1} \rightarrow K^{*}$ is an arbitrary homomorphism.

The remaining statements are straightforward.

2.1.2. Direct product. By definition, the ring of algebraic functions of $T\left(H_{1}, \alpha_{1}\right) \times T\left(H_{2}, \alpha_{2}\right)$ is the tensor product of the respective rings. We can write

$$
T\left(H_{1}, \alpha_{1}\right) \times T\left(H_{2}, \alpha_{2}\right)=T\left(H_{1} \oplus H_{2}, \alpha_{1} \oplus \alpha_{2}\right)
$$

by identifying

$$
e_{H_{1}, \alpha_{1}}\left(h_{1}\right) \otimes e_{H_{2}, \alpha_{2}}\left(h_{2}\right)=e_{H_{1} \oplus H_{2}, \alpha_{1} \oplus \alpha_{2}}\left(\left(h_{1}, h_{2}\right)\right) .
$$

2.1.3. Some standard morphisms. (i) Shifts. Any point $b \in T(H, 1)(K)=$ $\operatorname{Hom}\left(H, K^{*}\right)$ determines an automorphism $b^{*}$ of $T(H, \alpha)$ :

$$
b^{*}(e(h)):=h(b) e(h)
$$

where from now on we denote by $h(b)$ the value of $e_{H, 1}(h)$ at the point $b$.

(ii) Multiplication by $n$. This is the morphism

$$
[n]: T(H, \alpha) \rightarrow T\left(H, \alpha^{n^{2}}\right)
$$

defined by

$$
[n]^{*}\left(e_{H, \alpha^{n^{2}}}(h)\right)=e_{H, \alpha}(n h) .
$$

For $n=-1$ it is an endomorphism of $T(H, \alpha)$. It is also an endomorphism, if $\alpha$ takes values in roots of unity of degree $d$ and $n^{2} \equiv 1 \bmod d$.

The commutation rule with shifts is

$$
b^{*} \circ[n]^{*}=[n]^{*} \circ(n b)^{*} .
$$

(iii) External multiplication. It is the morphism

$$
m_{\alpha, \beta}: T(H, \alpha) \times T(H, \beta) \rightarrow T(H, \alpha \beta)
$$


defined by

$$
m_{\alpha, \beta}^{*}\left(e_{H, \alpha \beta}(h)\right)=e_{H, \alpha}(h) \otimes e_{H, \beta}(h) .
$$

(iv) Mumford's morphism. This is the morphism

$$
\begin{gathered}
M: T(H \oplus H, \alpha \oplus \alpha) \rightarrow T\left(H \oplus H, \alpha^{2} \oplus \alpha^{2}\right), \\
M^{*}(e(h, g))=e(h+g, h-g) .
\end{gathered}
$$

It is well defined, because

$$
\begin{gathered}
(\alpha \oplus \alpha)\left[(h+g, h-g),\left(h^{\prime}+g^{\prime}, h^{\prime}-g^{\prime}\right)\right] \\
=\alpha\left(h+g, h^{\prime}+g^{\prime}\right) \alpha\left(h-g, h^{\prime}-g^{\prime}\right)=\alpha^{2}\left(h, h^{\prime}\right) \alpha^{2}\left(g, g^{\prime}\right) .
\end{gathered}
$$

2.2. Periods. We choose and fix an abelian group of periods $B \subset T(H, 1)(K)$. The period group is written additively; it acts upon $T(H, \alpha)$ by shifts.

Trying to make sense of the quotient $T(H, \alpha) / B$ we will study formal or analytic functions on $T(H, \alpha)$ with automorphic properties with respect to the the group $\left\{b^{*} \mid b \in B\right\}$.

2.3. Definition. A (two-sided) theta multiplier $\mathcal{L}$ for the non-commutative torus $T(H, \alpha)$ and period group $B$ consists of the data $\mathcal{L}=\left(h_{l}, h_{r}, \psi,(),\right)$ where

(i) $h_{l}, h_{r}: B \rightarrow H$ are two group homomorphisms.

We also put $h^{ \pm}:=h_{l} \pm h_{r}$ and denote the image of $b \in B$ with respect to $h_{l}$ (resp. $\left.h_{r}, h^{ \pm}\right)$as $h_{b, l}$ (resp. $\left.h_{b, r}, h_{b}^{ \pm}\right)$.

(ii) $\psi: B \rightarrow K^{*}$ is also a group homomorphism.

(iii) (, ) : $B \times B \rightarrow K^{*}$ is a symmetric pairing.

These data must satisfy the following condition: for all $b_{1}, b_{2} \in B$

$$
h_{b_{2}}^{-}\left(b_{1}\right)=\left(b_{1}, b_{2}\right)^{2} \alpha\left(h_{b_{1}, l}, h_{b_{2}, l}\right) \alpha\left(h_{b_{1}, r}, h_{b_{2}, r}\right)^{-1}
$$

2.3.1. Remark. Moduli space of quotients $T(H, \alpha) / B$ locally splits into a product of the classical moduli space of commutative tori $T(H, 1) / B$ and the space of quantization parameters $\alpha$ (which in a sense also are "hidden periods": cf. our discussion of Connes' treatment of bad equivalence relations).

When $K=\mathbf{C}$, existence of sufficiently many theta functions is equivalent to the algebraicity of $T(H, 1) / B$ which becomes an abelian manifold. Multipliers of such theta functions satisfy Riemann symmetry and positivity conditions. 
Relations (2.12) represent an extension of the symmetry conditions to our enlarged moduli space. For a quantum version of positivity conditions, see Theorem $2.6 .1 \mathrm{~b}$ ) below.

2.4. Automorphy factors. For any theta multiplier $\mathcal{L}$ and period $b \in B$, the automorphy factor $j_{\mathcal{L}}(b)$ is, by definition, the following linear endomorphism of any of the function spaces $A l, A f, A n$ of $T(H, \alpha)$ :

$$
j_{\mathcal{L}}(b): \Phi \mapsto \psi(b)(b, b) e\left(h_{b, l}\right) \Phi e\left(h_{b, r}\right)^{-1} .
$$

Clearly, it is invertible.

2.5. Proposition. For $\mathcal{L}$ fixed, the map $b \mapsto j_{\mathcal{L}}(b)^{-1} \circ b^{*}$ is a group homomorphism.

It is injective if $\mathrm{Ker} h^{-}=0$.

Proof. We must check that

$$
j_{\mathcal{L}}\left(b_{1}+b_{2}\right)^{-1} \circ\left(b_{1}+b_{2}\right)^{*}=j_{\mathcal{L}}\left(b_{1}\right)^{-1} \circ b_{1}^{*} \circ j_{\mathcal{L}}\left(b_{2}\right)^{-1} \circ b_{2}^{*}
$$

We have in view of $(2.13),(2.4),(2.5)$ :

$$
j_{\mathcal{L}}(b)^{-1}(e(h))=\psi(b)^{-1}(b, b)^{-1} \varepsilon\left(h_{b, l}\right) \alpha\left(h, h_{b}^{+}\right) \alpha\left(h_{b, r}, h_{b, l}\right) e\left(h-h_{b}^{-}\right) .
$$

Now apply both sides of (2.14) to the arbitrary $e(h)$ and then compare them using (2.12). A somewhat lengthy but straightforward calculation gives (2.14).

When $h^{-}$is injective, $h_{b}^{-} \neq 0$ for non-zero $b$, so that $j_{\mathcal{L}}(b)^{-1} \circ b^{*}(e(h)) \neq e(h)$.

2.6. Theta functions and theta types. A (quantized) theta function with multiplier $\mathcal{L}$ is a formal series $\theta \in A f(T(H, \alpha))$ invariant with respect to the transformation group

$$
\left\{j_{\mathcal{L}}(b)^{-1} \circ b^{*} \mid b \in B\right\} .
$$

In other words, $\theta$ must satisfy the functional equations

$$
b^{*}(\theta)=\psi(b)(b, b) e\left(h_{b, l}\right) \theta e\left(h_{b, r}\right)^{-1}
$$

for all $b \in B$. Clearly, theta functions with multiplier $\mathcal{L}$ form a linear space which we denote $\Gamma(\mathcal{L})$. This notation is supposed to remind the case of usual abelian varieties where we deal with invertible sheaves and their sections.

Actually, different multipliers may produce the same space of theta functions or even homomorphisms $b \mapsto j_{\mathcal{L}}(b)^{-1} \circ b^{*}$. Consider, for example, the case of a commutative torus, where $\alpha \equiv 1$. Then $\Gamma(\mathcal{L})$ depends on $h_{l}, h_{r}$ only via their 
difference $h^{-}=h_{l}-h_{r}$. Moreover, if $(,)^{\prime}$ is another symmetric pairing such that $\varphi\left(b_{1}, b_{2}\right):=\left(b_{1}, b_{2}\right)^{\prime}\left(b_{1}, b_{2}\right)^{-1}$ takes values in $\{ \pm 1\}$, then $\varphi(b, b)$ is multiplicative in $b$, and we may replace $(\psi,()$,$) by \left(\psi^{\prime},(,)^{\prime}\right)$ where $\psi^{\prime}(b)=\phi(b, b) \psi(b)$.

Generally, from (2.15) one sees that if $j_{\mathcal{L}^{\prime}}(b)^{-1} \circ b^{*}=j_{\mathcal{L}}(b)^{-1} \circ b^{*}$ for all $b$, then $h^{-}=h^{\prime-}$ and moreover,

$$
\begin{gathered}
(b, b)^{-1} \alpha\left(h_{b, r}, h_{b, l}\right)= \pm(b, b)^{\prime-1} \alpha\left(h_{b, r}^{\prime}, h_{b, l}^{\prime}\right), \\
\psi(b)^{-1} \alpha\left(h, h_{b}^{+}\right)= \pm \psi^{\prime}(b)^{-1} \alpha\left(h, h_{b}^{\prime+}\right) .
\end{gathered}
$$

We will call two multipliers equivalent, if they have the same space of thetafunctions. (This definition is reasonable only for ample multipliers, see below). An equivalence class of multipliers $L$ will be called a theta type. The space $\Gamma(\mathcal{L})$ depends only on this class and can be denoted also $\Gamma(L)$.

2.6.1. Theorem. a) We have

$$
\operatorname{dim} \Gamma(\mathcal{L})=\left[H: h^{-}(B)\right]
$$

b) Assume that $K$ is a normed field. Then all theta functions of type $\mathcal{L}$ are analytic if $\left[H: h^{-}(B)\right]<\infty$ and

$$
\log \left|(b, b) \alpha\left(h_{b, l},-h_{b, r}\right)\right|
$$

is a positively defined quadratic form on $B$.

In particular, assume that $B$ is free and the quantization parameter $\alpha$ is unitary, $\left|\alpha\left(h, h^{\prime}\right)\right| \equiv 1$. Then this condition means that $\operatorname{rk} B=\operatorname{rk} H, B$ is discrete in $T(H, 1)(K)$ and $\log |(b, b)|$ is positively defined.

Proof. Let $\theta=\sum_{h \in H} a_{h} e(h), a_{h} \in K, b \in B$. We have

$$
b^{*}(\theta)=\sum_{h \in H} a_{h} h(b) e(h)=\sum_{h \in H} a_{h+h_{b}^{-}}\left(h+h_{b}^{-}\right)(b) e\left(h+h_{b}^{-}\right),
$$

whereas the right hand side of (2.16) is:

$$
\psi(b)(b, b) \sum_{h \in H} a_{h} \varepsilon\left(h_{b, r}\right) \alpha\left(h_{b, r}, h_{b, i}\right) \alpha\left(h_{b}^{+}, h\right) e\left(h+h_{b}^{-}\right) .
$$

In (2.19) we replace $h_{b}^{-}(b)$ by $(b, b)^{2} \varepsilon\left(h_{b, l}\right) \varepsilon\left(h_{b, r}\right)$ (see $(2.12)$ ). Comparing coefficients of (2.19) and (2.20), we see that (2.16) is equivalent to

$$
a_{h+h_{b}^{-}}=a_{h} \psi(b) h(b)^{-1}(b, b)^{-1} \varepsilon\left(h_{b, l}\right) \alpha\left(h_{b, r}, h_{b, l}\right) \alpha\left(h_{b}^{+}, h\right)
$$


for all $h \in H$ and $b \in B$. Thus one can arbitrarily choose values $a_{h}$ for all $h$ in a system of representatives of $H / h^{-}(B)$ and then uniquely reconstruct $\theta$. This proves the first statement of the theorem. It also shows that if $\Gamma(\mathcal{L})$ is not finite dimensional, it necessarily contains non-analytic functions.

Assume now that $\Gamma(\mathcal{L})$ is finite dimensional. Then on each coset $h+h^{-}(B)$ we have

$$
\log \left|a_{h+h_{b}^{-}}\right|=\log \left|a_{h}\right|-\log \left|(b, b) \alpha\left(h_{b, r}, h_{b, l}\right)\right|+\log \left|\psi(b) h(b)^{-1} \alpha\left(h_{b}^{+}, h\right)\right| .
$$

The second summand in the right hand side is quadratic in $b$ whereas the third is linear. Hence analyticity follows from the positive definiteness of (2.18).

2.6.2. Multiplication of theta functions. Generally, we can multiply analytic functions, but not formal ones. We will call a theta multiplier $\mathcal{L}$ analytic, if $\Gamma(\mathcal{L})$ consists of analytic functions, and ample, if $\mathcal{L}$ satisfies conditions of Theorem $2.6 .1 \mathrm{~b})$.

We will call two analytic theta multipliers $\mathcal{L}_{i}=\left(h_{l}^{(i)}, h_{r}^{(i)}, \psi_{i},(,)_{i}\right), i=1,2$, composable (in this order) if $h_{l}^{(2)}=h_{r}^{(1)}$. Define their product as

$$
\mathcal{L}_{1} \otimes \mathcal{L}_{2}=\mathcal{L}:=\left(h_{l}^{(1)}, h_{r}^{(2)}, \psi_{1} \psi_{2},(,)_{1}(,)_{2}\right)
$$

A straightforward calculation shows that if $\mathcal{L}_{i}$ are ample, $\mathcal{L}$ is ample as well, and the product of theta functions produces a well defined map

$$
\Gamma\left(\mathcal{L}_{1}\right) \otimes \Gamma\left(\mathcal{L}_{2}\right) \rightarrow \Gamma(\mathcal{L}): \theta_{1} \otimes \theta_{2} \mapsto \theta_{1} \theta_{2}
$$

One can call two ample theta types $L_{1}$ composable, if they contain pairs of composable multipliers. The multiplication in (2.22) does not depend on the choice of such a pair, but I did not check that the product type $L$ cannot change.

2.6.3. Quantized abelian varieties. Assume that $(T(H, \alpha), B)$ admits ample theta multipliers. Then we consider $\oplus_{L} \Gamma(L)$ where $L$ runs over all theta types with $\psi(b) \in\{ \pm 1\}$, together with partial multiplication defined above, as a quantized version of the graded coordinate ring of an abelian variety. In the classical case, it is graded by the symmetric elements of Pic lying in the effective cone (see 2.7.2 below).

2.7. Functorial properties of theta functions. Consider a morphism of non-commutative tori $F: T\left(H_{2}, \alpha_{2}\right) \rightarrow T\left(H_{1}, \alpha_{1}\right)$. As in Proposition 2.1.1, let $F^{*}(e(h))=a_{h} e(f(h)), a_{h} \in K^{*}, f: H_{1} \rightarrow H_{2}$. The map $f$ induces a morphism of commutative tori which we also denote $F: T\left(H_{2}, 1\right) \rightarrow T\left(H_{1}, 1\right)$. Let $B_{i} \subset$ $T\left(H_{i}, 1\right)(K)$ be two period lattices such that $F\left(B_{2}\right) \subset B_{1}$. 
Choose a theta multiplier $\mathcal{L}_{1}=\left(h_{l}, h_{r}, \psi,(),\right)$ for $T\left(H_{1}, \alpha_{1}\right)$ and $B_{1}$. We will show, how to produce from it a new theta multiplier

$$
\mathcal{L}_{2}=F^{*}\left(\mathcal{L}_{1}\right)=\left(h_{l}^{\prime}, h_{r}^{\prime}, \psi^{\prime},(,)^{\prime}\right)
$$

for $T\left(H_{2}, \alpha_{2}\right), B_{2}$, if the following condition holds:

the characteristic of $F$ is 1 , that is, $a_{h+g}=a_{h} a_{g}$ (cf. end of the proof of Proposition 2.2.1), and $f$ is compatible with $\alpha_{1}$ and $\alpha_{2}$, and not just their squares.

The map $f: H_{1} \rightarrow H_{2}$ induces a map $F: \operatorname{Hom}\left(H_{2}, K^{*}\right) \rightarrow \operatorname{Hom}\left(H_{1}, K^{*}\right)$ whose restriction on $B_{2}$ sends it to $B_{1}$. Put for $b, b_{1}, b_{2} \in B_{2}$ :

$$
h_{l, r}^{\prime}=f \circ h_{l, r} \circ F: B_{2} \rightarrow H_{2}
$$

so that $h_{b, l}^{\prime}=f\left(h_{F(b), l}\right)$ and similarly for $h_{r}^{\prime}, h^{\prime \pm}$,

$$
\begin{gathered}
\psi^{\prime}(b)=\psi(F(b)) a_{h_{F(b)}^{-}}, \\
\left(b_{1}, b_{2}\right)^{\prime}=\left(F\left(b_{1}\right), F\left(b_{2}\right)\right) .
\end{gathered}
$$

2.7.1. Theorem. The data (2.23)-(2.25) constitute a theta multiplier for $\mathrm{T}\left(\mathrm{H}_{2}, \alpha_{2}\right)$, $B_{2}$ such that $F^{*}(\Gamma(\mathcal{L})) \subset \Gamma\left(F^{*}(\mathcal{L})\right)$.

Proof. We have first to check that $(2.12)$ holds for $F^{*}(\mathcal{L})$. For $b_{1}, b_{2} \in B_{2}$, the left hand side becomes

$$
h_{b_{2}}^{\prime-}\left(b_{1}\right)=f\left(h_{F\left(b_{2}\right)}^{-}\right)\left(b_{1}\right)=h_{F\left(b_{2}\right)}^{-}\left(F\left(b_{1}\right)\right) .
$$

Furthermore, if the characteristic of $F$ is 1 , the right hand side can be rewritten as

$$
\begin{gathered}
\left(b_{1}, b_{2}\right)^{\prime 2} \alpha_{2}\left(f\left(h_{F\left(b_{1}\right), l}\right), f\left(h_{F\left(b_{2}\right), l}\right)\right) \alpha_{2}\left(f\left(h_{F\left(b_{1}\right), r}\right), f\left(h_{F\left(b_{2}\right), r}\right)\right)^{-1} \\
=\left(F\left(b_{1}\right), F\left(b_{2}\right)\right)^{2} \alpha_{1}\left(h_{F\left(b_{1}\right), l}, h_{F\left(b_{2}\right), l}\right) \alpha_{1}\left(h_{F\left(b_{1}\right), r}, h_{F\left(b_{2}\right), r}\right)^{-1} .
\end{gathered}
$$

Applying (2.12) to $F\left(b_{1}\right), F\left(b_{2}\right)$ in lieu of $b_{1}, b_{2}$, we see that both expressions coincide.

Let us now check that $F^{*}(\Gamma(\mathcal{L})) \subset \Gamma\left(F^{*}(\mathcal{L})\right)$.

Choose a formal function

$$
\theta=\sum_{h \in H_{1}} c_{h} e(h), e=e_{H_{1}, \alpha_{1}}
$$


According to $(2.21)$, it belongs to $\Gamma(\mathcal{L})$ iff the following conditions are satisfied for all $h \in H_{1}, b \in B_{1}$ :

$$
c_{h+h_{b}^{-}}=c_{h} \psi(b) h(b)^{-1}(b, b)^{-1} \varepsilon_{1}\left(h_{b, l}\right) \alpha_{1}\left(h_{b, r}, h_{b, l}\right) \alpha_{1}\left(h_{b}^{+}, h\right) .
$$

Notice that our notation slightly differs from $(2.21): a_{h}$ is now reserved for $F^{*}(e(h))=$ $a_{h} e(f(h))$ as in the first lines of 2.7 .

Put now

$$
F^{*}(\theta)=\sum_{g \in H_{2}} C_{g} e(g), \quad e=e_{H_{2}, \alpha_{2}} .
$$

We have $C_{g}=0$, if $g \notin f\left(H_{1}\right)$; otherwise

$$
C_{g}=\sum_{h \in f^{-1}(g)} c_{h} a_{h}
$$

To prove that $F^{*}(\theta) \in \Gamma\left(F^{*}(\mathcal{L})\right)$, we will check that $C_{g}$ satisfy analogs of relations (2.26) written for all $g \in H_{2}$ and all $b \in B_{2}$.

Consider first the case $g \notin f\left(H_{1}\right)$. Then the relevant analog of $(2.26)$ says that $C_{g+h_{b}^{\prime-}}$ must be proportional to $C_{g}$ that is, zero. This is indeed true, because in view of $(2.23), h_{b}^{\prime-}=f\left(h_{F(b)}^{-}\right)$and thus $g+h_{b}^{\prime-} \notin f\left(H_{1}\right)$.

Now assume that $g \in f\left(H_{1}\right)$, fix also $b \in B_{2}$, and write down separately both sides of the relevant case of (2.26). Since characteristic of $F$ is 1 , the left hand side can be written as

$$
\begin{gathered}
C_{g+h_{b}^{\prime-}}=\sum_{h \in f^{-1}(g)} c_{h+h_{F(b)}^{-}} a_{h} a_{h_{F(b)}^{-}} \\
=\sum_{h \in f^{-1}(g)} c_{h} a_{h} \psi(F(b)) a_{h_{F(b)}^{-}} h(F(b))^{-1}(F(b), F(b))^{-1} \\
\times \varepsilon_{1}\left(h_{F(b), l}\right) \alpha_{1}\left(h_{F(b), r}, h_{F(b), l}\right) \alpha_{1}\left(h_{F(b)}^{+}, h\right) .
\end{gathered}
$$

Here we have rewritten $c_{h+h_{F(b)}^{-}}$using (2.26).

The right hand side is

$$
\left(\sum_{h \in f^{-1}(g)} c_{h} a_{h}\right) \psi^{\prime}(b) g(b)^{-1}(F(b), F(b))^{-1} \varepsilon_{2}\left(h_{b, l}^{\prime-}\right) \alpha_{2}\left(h_{b, r}^{\prime-}, h_{b, l}^{\prime-}\right) \alpha_{2}\left(h_{b}^{\prime+}, g\right) .
$$

We can now compare (2.28) and (2.29) term by term using (2.23)-(2.25), and convince ourselves that they coincide. In particular, we use the identities $g(b)=$ $h(F(b))$ and $g=f(h)$ for any $h \in f^{-1}(g)$. 
2.7.2. Examples. All morphisms of tori described in 2.1 .3 have characteristic 1. Therefore, complementing these tori by compatible period lattices, we obtain quantized versions of many standard morphisms of abelian varieties.

In particular, $[-1]^{*}$ acts on theta multipliers for $T(H, \alpha), B$ by simply inverting $\psi$. Hence symmetric theta multipliers correspond to $\psi: B \rightarrow\{ \pm 1\}$.

Mumford's morphism for abelian varieties, induced by the toric morphism (2.11), is the starting point for his study of homogeneous coordinate rings of abelian varieties. Our preparatory work allows us to hope that at least part of this study can be extended to the quantum case.

\section{Appendix. Commutative Geometry as Noncommutative Geometry}

The main goal of this Appendix is to illustrate Connes approach to noncommutative geometry in an algebraic geometric context and to make convincing our claim that an elliptic curve "is" a two-dimensional noncommutative torus.

We take as our starting point Connes explanations about how to treat as a noncommutative space quotient of a "commutative space" by an equivalence relation or equivalence groupoid: see [Co], II.2-II.5. This viewpoint is complementary to the more popular in algebraic geometry deformation paradigm.

First, a reminder about groupoids.

Let $U$ be a set. Classically, an equivalence relation $\sim$ on $U$ is given by its graph $R \subset U \times U, R:=\{(a, b) \mid a \sim b\}$ which satisfies the three conditions:

Reflexivity:

$$
a \sim a \Longleftrightarrow \Delta_{U} \subset R
$$

Symmetry:

$$
(a \sim b \Leftrightarrow b \sim a) \Longleftrightarrow s_{12}(R)=R
$$

Transitivity:

$$
((a \sim b) \&(b \sim c) \Rightarrow a \sim c) \Longleftrightarrow \operatorname{pr}_{13}[(R \times U) \cap(U \times R)] \subset R
$$

All of this can be rephrased as follows: there exists a category with the set of objects $U$, set of morphisms $R$, such that $R \rightarrow U \times U$ is the map $f \mapsto$ (source of $f$, target of $f$ ), and in addition, every morphism is an isomorphism, and all automorphism groups are trivial.

Consider now a diagram $R \rightarrow U \times U$ satisfying this description with the last condition deleted so that the automorphism groups can now be arbitrary. We will call such a diagram an equivalence groupoid (on the set $U$ ). Of course, an 
equivalence groupoid $R \rightarrow U \times U$ comes together with the identity map $U \rightarrow R$ : $a \mapsto \mathrm{id}_{a}$ and the associative multiplication map $R \times_{U} R \rightarrow R$ satisfying the usual categorical axioms which reduce to the reflexivity, symmetry, and transitivity for the usual equivalence relations. Notice that the image of $R$ is in fact an equivalence relation, and the respective quotient is the set of isomorphism classes of objects.

Thus the basic difference between equivalence groupoids and equivalence relations on sets can be demonstrated on one-point sets $U=\{*\}$ : in this case $R$ is simply a group. In the framework of homotopy theory, the respective quotient object $\{*\} / R$ is represented by the classifying space $B R$. Stacks provide a categorical context for constructing such quotients in algebraic geometry.

In fact, the notion of equivalence groupoid was formulated in such a way that it readily generalizes to the case when $R \rightarrow U \times U$ is a diagram in an arbitrary category with products, e.g. schemes. One should imagine $U$ as an atlas and $R$ as gluing rules, so that the geometric object we are interested in is symbolically $U / R$.

Connes prescription, roughly speaking, consists in reducing geometric study of $X=U / R$ to the representation theory of a non-commutative ring $\mathcal{R}_{X}$. The heuristic rule for constructing $\mathcal{R}_{X}$ can be stated as follows. Let us write elements (points) of $R$ as morphisms $j: u \rightarrow u^{\prime}$ so that $R \rightarrow U \times U$ maps such a point to $\left(u, u^{\prime}\right)$. Then $\mathcal{R}_{X}$ consists of certain functions $f$ on $R$ endowed with convolution multiplication:

$$
(f * g)\left(k: u \rightarrow u^{\prime \prime}\right)=\sum_{(i, j): i j=k} f\left(j: u \rightarrow u^{\prime}\right) g\left(i: u^{\prime} \rightarrow u^{\prime \prime}\right) .
$$

Of course, correct choice of the class of functions and making sense of the convolution multiplication may present a problem, but in algebraic geometry it is clear what to start with at least when $U$ is affine.

Let us illustrate such a setup by several examples.

Example 1. If $X$ is the quotient $\{*\} / G$ where $G$ is a finite group, then $\mathcal{R}_{X}$ is the group ring of $G$. Representation theory of $\mathcal{R}_{X}$ is essentially $K$-theory of the classifying stack of $G$, in accordance with the common wisdom.

Example 2. If $X$ is the quotient of an affine scheme $U=\operatorname{Spec} A$ by the action of a group $G$, then $\mathcal{R}_{X}$ should contain at least the twisted product of $A$ with a version of the group ring of $G$.

When $U=\mathbf{G}_{m}$ with coordinate $y$ and $G=\mathbf{Z}$ with generator $x$ acting on $y$ as multiplication by $q$, then in this twisted product we have $x y x^{-1}=q x$ that is, the basic relation of the noncommutative torus. On the other hand, $U / G$ makes sense as an elliptic curve in analytic geometry, if $|q| \neq 1$.

Example 3. A projective scheme $\operatorname{Proj} A$ is the quotient of its cone $\operatorname{Spec} A$ (with vertex deleted) by the action of $\mathbf{G}_{m}$ determining the grading. Studying representations of the twisted product of $A$ with the group ring of $G_{m}$ is equivalent to studying 
graded $A$-modules. Deleting the vertex boils down to taking the quotient of the category of graded modules by the subcategory of modules with finite number of nonvanishing components. This is the familiar Serre's picture which is archetypal in the following sense: after finding an appropriate ring $\mathcal{R}_{X}$, one proceeds to establish an equivalence of categories $\mathcal{R}_{X}-\mathrm{Mod} \rightarrow \mathrm{Coh}_{X}$, or its localized and/or derived version.

\section{References}

[AP] D. Arinkin, A. Polishchuk. Fukaya category and Fourier transform. Preprint math.AG/9811023

[Bar] S. Barannikov. Extended moduli spaces and mirror symmetry in dimensions $n>3$. Preprint math.AG/9903124

[BarK] S. Barannikov, M. Kontsevich. Frobenius manifolds and formality of Lie algebras of polyvector fields. Int. Math. Res. Notices, 4 (1998), 201-215.

[BEG] V. Baranovsky, S. Evens, V. Ginzburg. Representations of quantum tori and double-affine Hecke algebras. Preprint math.RT/0005024

[Bat] V. Batyrev. Dual polyhedra and the mirror symmetry for Calabi-Yau hypersurfaces in toric varieties. Journ. Alg. Geom., 3 (1994), 493-535.

[Co] A. Connes. Noncommutative geometry. Academic Press, 1994.

[De] P. Deligne. Local behavior of Hodge structures at infinity. In: Mirror Symmetry II, ed. by B. Greene and S. T. Yau, AMS-International Press, 1996, 683-699.

[Fu] K. Fukaya. Mirror symmetry of abelian variety and multi theta functions. Preprint, 1998.

[Giv] A. Givental. Equivariant Gromov-Witten invariants. Int. Math. Res. Notes, 13 (1996), 613-663.

[GolLO] V. Golyshev, V. Lunts, D. Orlov. Mirror symmetry for abelian varieties. Preprint math.AG/9812003

[Gr1] M. Gross. Special Lagranfian fibrations I: Topology. alg-geom/9710006

[Gr2] M. Gross. Special Lagranfian fibrations II: Geometry. math.AG/9809072

[Ko] M. Kontsevich. Homological algebra of Mirror Symmetry. Proceedings of the ICM (Zürich, 1994), vol. I, Birkhäuser, 1995, 120-139. Preprint alggeom/9411018.

[LYZ] N. C. Leung, Sh.-T. Yau, E. Zaslow. From special Lagrangian to HermitianYang-Mills via Fourier-Mukai transform. Preprint math.DG/0005118.

[Ma1] Yu. Manin. Quantized theta-functions. In: Common Trends in Mathematics and Quantum Field Theories (Kyoto, 1990), Progress of Theor. Phys. Supplement, 102 (1990), 219-228. 
[Ma2] Yu. Manin. Frobenius manifolds, quantum cohomology, and moduli spaces. AMS Colloquium Publications, vol. 47, Providence, RI, 1999, xiii+303 pp.

[Ma3] Yu. Manin. Moduli, Motives, Mirrors. Plenary talk at 3rd European Congress of Mathematicians, Barcelona, 2000, Preprint.

[MirS1] S.-T. Yau, ed. Essays on Mirror Manifolds. International Press Co., Hong Cong, 1992.

[MirS2] B. Greene, S. T. Yau, eds. Mirror Symmetry II., AMS-International Press, 1996.

$[\mathrm{Mu}]$ D. Mumford. On the equations defining abelian varieties I. Inv. Math. 1 (1966), 355-374.

[Po1] A. Polishchuk. Massey and Fukaya products on elliptic curve. Preprint math.AG/9803017

[Po2] A. Polishchuk. Homological mirror symmetry with higher products. Preprint math.AG/9901025

[PoZ] A. Polishchuk, E. Zaslow. Categorical mirror symmetry: the elliptic curve. Adv. Theor. Math. Phys., 2 (1998), 443-470. Preprint math.AG/9801119

[RiS] M. A. Rieffel, A. Schwarz. Morita equivalence of multidimensional tori. Preprint math.QA/9803057

[So] Y. Soibelman. Quantum tori, mirror symmetry and deformation theory. Preprint, 2000.

[StYZ] A. Strominger, S.-T.Yau, E. Zaslow. Mirror symmetry is T-duality. Nucl. Phys. B 479 (1996), 243-259.

[We] A. Weinstein. Classical theta functions and quantum tori. Publ. RIMS, Kyoto Univ., 30 (1994), 327-333. 